\title{
CONFORTO ACÚSTICO DE ESCRITÓRIOS EM AMBIENTE INDUSTRIAL DE USINA HIDRELÉTRICA
}

\section{ACOUSTICAL COMFORT OF OFFICES IN INDUSTRIAL ENVIRONMENT OF HYDROELECTRIC POWER PLANT}

\author{
Rodrigo Valdeci Martins ${ }^{1}$ \\ Universidade Federal de Santa Catarina, Florianópolis, SC, Brasil, rodrigovmartins@outlook.com \\ Erasmo Felipe Vergara Miranda ${ }^{2}$ \\ Universidade Federal de Santa Catarina, Florianópolis, SC, Brasil, e.f.vergara@ufsc.br
}

\begin{abstract}
Resumo
A clareza na comunicação, o conforto e a concentração da atenção são fundamentais no desempenho e produtividade dos profissionais que utilizam escritórios em ambientes industriais. A avaliação do conforto acústico nesses escritórios deve considerar a medição de níveis de pressão sonora (NPS), de tempos de reverberação (TR), bem como a quantificação do ruído de fundo e da inteligibilidade da fala. A presente pesquisa teve como objetivo avaliar o conforto acústico de ambientes de escritórios situados na casa de força de uma usina hidrelétrica. Para tanto, a investigação baseou-se na avaliação da interferência do ruído de fundo e do TR na inteligibilidade da fala em seis ambientes de três escritórios, a partir de medições de NPS, da quantificação e análise das curvas critério de ruído NC e RC Mark II, e do Índice de Transmissão da Fala (STI). Os seis ambientes internos também foram avaliados pelo cálculo do TR de Sabine e Eyring. Todos os ambientes não atenderam ao critério de conforto da curva NC 40, apresentando espectros desbalanceados entre 125 e $500 \mathrm{~Hz}$ e vibrações perceptíveis nas baixas frequências ( 16 ou $31,5 \mathrm{~Hz}$ ) que superaram os $75 \mathrm{~dB}$. Quatro ambientes atenderam aos valores de TR recomendados, mantendo-se entre 0,3 e 0,5 segundo. O STI foi classificado entre ruim e razoável, variando entre 0,2 e 0,5. Conclui-se que ambientes industriais como casas de força de usinas hidrelétricas semelhante as estudadas não favorecem a instalação de escritórios sem interferência no conforto acústico e na comunicação oral dos usuários, sendo necessário um projeto adequado para utilização prolongada em trabalhos intelectuais.
\end{abstract}

Palavras-chave: Conforto acústico de escritórios. Ambiente industrial. Interferência na fala.

\begin{abstract}
The clarity in communication, comfort, and concentration are critical issues in the performance and productivity of professionals who use offices in industrial environments. The evaluation of acoustic comfort in these offices should consider the measurement of sound pressure levels (SPL), reverberation times (RT), as well as the quantification of background noise and speech intelligibility. The present research aims to evaluate acoustic comfort in office environments located in the powerhouse of a hydroelectric plant. For this, the investigation was based on the evaluation of the interference of background noise and TR in speech intelligibility of six office rooms from three office buildings, based on NPS measurements, quantification and analysis of the NC and RC Mark II noise criterion curves, and the Speech Transmission Index (STI). The six indoor environments were also evaluated through the calculation of the TR of Sabine and Eyring. All office rooms did not meet the comfort criteria of the NC 40 curve, had unbalanced spectra between 125 and $500 \mathrm{~Hz}$ and perceptible vibrations at low frequencies ( 16 or $31.5 \mathrm{~Hz}$ ) that exceeded $75 \mathrm{~dB}$. Four rooms attended recommended TR values, remaining between 0.3 and 0.5 seconds. The STI was rated between bad and reasonable, ranging from 0.2 to 0.5 . It can be concluded that industrial environment as powerhouses of hydroelectric plants similar to those studied does not favor the installation of offices without interfering with the acoustic comfort and oral communication of the users, being necessary an adequate project for prolonged use in intellectual works.
\end{abstract}

Keywords: Acoustic comfort of offices. Industrial environment. Speech interference.

\section{How to cite this article:}

MARTINS, R.C.; MIRANDA, E.F.V. Conforto acústico de escritórios em ambiente industrial de usina hidrelétrica. PARC Pesquisa em Arquitetura e Construção, Campinas, SP, v. 10, p. e0190X, 2019. ISSN 1980-6809

DOI:http://dx.doi.org/10.20396/parc.v10i0.8653712 


\section{Introdução}

Para um ambiente de trabalho, principalmente aqueles situados em ambientes industriais, além de requisitos básicos a serem atendidos durante o projeto (conforto térmico, conforto lumínico e ergonomia), a elaboração de projeto acústico se faz necessária (ISO, 1996b; HUANG et al., 2011; PASSERO; ZANNIN, 2012a; PIERRETTE et al., 2015; AL HORR et al., 2016; SELAMAT; ZULKIFLI, 2016; LIU et al., 2017). Uma grande quantidade de trabalhos tem como tema o conforto acústico de escritórios, contudo poucos abordam o caso de escritórios em ambientes industriais.

Ruídos no ambiente de escritório causam desmotivação, fadiga, estresse, níveis baixos de privacidade e concentração, afetando o desempenho e produtividade dos trabalhadores (HEDGE, 1982; EVANS; JOHNSON, 2000; VISCHER, 2007; JAHNCKE; HONGISTO; VIRJONEN, 2013; GOLMOHAMMADI; ALIABADI; NEZAMI, 2017). Segundo Golmohammadi, Aliabadi e Nezami (2017) a perda de concentração é a principal consequência gerada pelo ruído de fundo, principalmente nas tarefas em que é preciso se concentrar muito. São necessários aproximadamente oito minutos para que trabalhadores alcancem o mesmo nível de concentração após uma interrupção causada por ruído (KIM; DEAR, 2013). Por estes motivos, um condicionamento acústico criterioso do ambiente de trabalho se faz necessário. Contudo, este condicionamento pode ser uma tarefa complexa, pois devem ser consideradas interações entre diversas variáveis acústicas, tais como: absorção de materiais e mobiliário, nível de pressão sonora (NPS), dimensões dos ambientes, etc. (KERANEN; HONGISTO, 2013; HAAPAKANGAS et al., 2014). Reintem et al. (2017) enfatizam que os critérios relacionados aos níveis sonoros e à inteligibilidade da fala no interior de ambientes são determinantes no desempenho de tarefas humanas e na acústica de salas.

Diante ao exposto, a presente pesquisa tem como objetivo avaliar o conforto acústico em seis ambientes de três escritórios situados na casa de força de uma usina hidrelétrica. Para tanto, a investigação baseou-se na avaliação da interferência do ruído de fundo e do TR na inteligibilidade da fala, a partir de medições de NPS, da quantificação e análise das curvas critério de ruído NC e RC Mark II e do Índice de Transmissão da Fala (STI).

\section{Referencial teórico}

Diversos índices de ruído têm sido estipulados por vários autores com o intuito de descrever os múltiplos aspectos do ruído. De tempos em tempos e em diversos contextos cada um desses índices mostrou-se adequado para descrever sensações subjetivas da intensidade percebida, incômodo, fontes de ruído e características do ambiente. O NPS equivalente ponderado " $A$ " ( $\left(L A_{e q}\right)$ é, provavelmente, o índice mais difundido, pois se correlaciona bem com muitos efeitos psicofisiológicos do ruído (DELLA CROCIATA; MARTELLOTTA; SIMONE, 2012; DELLA CROCIATA; SIMONE; MARTELLOTTA, 2013). No entanto, diferentes índices também podem ser utilizados, como o NPS equivalente ( $\left.\mathrm{L}_{\mathrm{eq}}\right)$, o Noise Criterion (NC), o Preferred Noise Criterion (PNC), o Room Criterion (RC), o Balanced Noise Criterion (NCB), o Room Criterion Mark II (RC Mark II), o Quality Assessment Index (QAI) e o Nível de Interferência na Fala - Speech Interference Level (SIL) e Preferred Speech Interference Level (PSIL), os quais podem ser determinados a partir dos espectros medidos em campo (AYR; CIRILLO; MARTELLOTTA, 2001, 2002; DELLA CROCIATA; SIMONE; MARTELLOTTA, 2013). O índice PSIL é a média aritmética dos níveis de pressão sonora nas frequências centrais das bandas de oitava entre 500 e $2.000 \mathrm{~Hz}$ e proporciona as distâncias máximas nas quais se pode manter 
uma conversa inteligível, com voz normal ou muito alta, em função dos diferentes valores obtidos (ANSI/ASA, 1997; ISO, 1996a).

Os principais fatores que influenciam na resposta subjetiva das pessoas em relação ao ruído ambiente são a intensidade do ruído, seu potencial de interferência na atividade a ser desenvolvida no local e a qualidade do ruído de fundo. Assim, considerar estes fatores permite estimar correlações com medidas objetivas da avaliação do ruído no ambiente (GONZÁLEZ, 2008). Deste modo, o critério RC Mark Il é um método ideal para avaliar o ruído no interior de ambientes, uma vez que é multidimensional na sua composição e permite avaliar a intensidade relativa e a qualidade do ruído de fundo.

O estudo de Blazier jr (1981) sobre a avaliação acústica de ambientes pelas curvas RC originou as curvas RC Mark II, as quais foram posteriormente adotadas pela American Society of Heating, Refrigerating and Air-Conditioning Engineers (ASHRAE) a partir de 2001 (BLAZIER JR, 1995, 1997; ASHRAE, 2001). Foram incluídas modificações às curvas RC na banda de oitava de $16 \mathrm{~Hz}$, melhoras no procedimento da avaliação da qualidade acústica e o desenvolvimento de uma escala para estimar a magnitude da resposta subjetiva em função do desbalanceamento do espectro. O método RC Mark II fornece descritores da qualidade espectral (aproximações de respostas subjetivas ao ruído de fundo) e inclui um índice de avaliação de qualidade (QAI) que fornece uma estimativa da avaliação dos ocupantes, variando de aceitável a não aceitável. Este método tem sido aplicado em diversas situações para verificar os efeitos no desempenho das pessoas em ambientes laborais e sua percepção sob condições de ruído.

Wang e Bowden (2003) analisaram prós e contras dos critérios NC, NCB, RC e RC Mark II comparando e correlacionando os valores medidos em salas de aula com avaliações subjetivas da percepção humana. Bowden e Wang (2006) investigaram os efeitos de vários tipos de ruído de ar condicionado na produtividade e percepção de ocupantes de locais de trabalho e também examinaram três diferentes fases do ruído de fundo (faixa dos NPS, qualidade e características espectrais). A pesquisa de Ryherd e Wang (2008) mostrou as diferenças no desempenho de tarefas e na percepção de espectro de ruído de fundo e de ventilação com tonalidades distintas utilizando os critérios NC, BNC, RC, RC Mark II e LA eq.

O projeto de ambientes de trabalho deve considerar estratégias e medidas de controle do ruído, assim como prever os níveis de pressão sonora nesses locais (ISO, 1996b). O controle do ruído de fundo e do tempo de reverberação (TR) de salas pode beneficiar a qualidade acústica e a inteligibilidade da fala de um ambiente. $O$ isolamento acústico de escritórios privados (uma pessoa por sala), escritórios de plano aberto (mais de cinco pessoas por sala) e de escritórios compartilhados (duas ou mais pessoas por sala) cumpre um rol essencial no desenvolvimento das atividades laborais, na privacidade da fala e na tranquilidade no trabalho (KAARLELA-TUOMAALA et al., 2009; LEE, 2010; HONGISTO et al., 2016).

O estudo do conforto acústico em escritórios panorâmicos de Passero e Zannin (2009) revelou que os valores do NPS variaram de 64,9 a $67,2 \mathrm{~dB}(\mathrm{~A})$, principalmente nas bandas de frequência de oitava de 500 a $2.000 \mathrm{~Hz}$ (frequências típicas da fala humana). Além disso, os valores do TR foram de 1,25, 1,31 e 1,29 s nas bandas de frequências de oitava de $500,1.000$ e $2.000 \mathrm{~Hz}$, respectivamente. Passero e Zannin (2012a) também avaliaram acusticamente um escritório real com o objetivo de verificar a qualidade acústica e a influência de alguns materiais de construção no ruído ambiental e condicionamento acústico e concluíram que, para o escritório estudado ter boas condições acústicas, o material de acabamento da superfície do teto deveria ter alta absorção acústica, em média, superior a 0,8 . 
Golmohammadi, Aliabadi e Nezami (2017) avaliaram acusticamente estações de trabalho em espaços abertos de bancos segundo critérios de inteligibilidade da fala, medições do NPS em bandas de oitava $(125 \mathrm{a} 8.000 \mathrm{~Hz})$ e do TR. Para critérios de inteligibilidade da fala, nas bandas de oitava de 500 a $2.000 \mathrm{~Hz}$, foi utilizado o Preferred Noise Criterion (PNC) e constatou-se que os valores médios de 48,2 dB ultrapassaram os limites recomendados para o tipo de espaço estudado, o TR médio foi de 1,24 s e 95\% dos trabalhadores se incomodaram com os níveis de ruído de fundo.

Salas destinadas para fala requerem um TR pequeno. Para salas pequenas (até $200 \mathrm{~m}^{3}$ ) um valor de $0,5 \mathrm{~s}$ para a frequência de $500 \mathrm{~Hz}$ é considerado apropriado (MEHTA; JOHNSON; ROCAFORT, 1999). Os tempos de reverberação elevados tornam as salas acusticamente vivas, ou seja, a maior parte das ondas sonoras encontra anteparos e é refletida (permanecendo no ambiente e causando desconforto acústico nos usuários do espaço). Um ambiente com essas características dificulta muito a concentração para a realização de tarefas. Diminuindo-se os tempos de reverberação ou, em outras palavras, aumentando-se a absorção no interior do ambiente, cria-se um ambiente mais adequado ao trabalho, tanto pela maior facilidade na comunicação proporcionada pelos tempos de reverberação menores quanto pela diminuição dos níveis de pressão sonora (EGAN, 1988; MEHTA; JOHNSON; ROCAFORT, 1999; LONG, 2011).

Baseando-se no volume do espaço de estações de trabalho de bancos, Golmohammadi, Aliabadi e Nezami (2017) observaram tempos de reverberação médios de 1,15 s (volume $\leq 1.000 \mathrm{~m}^{3}$ ) e $1,34 \mathrm{~s}\left(\right.$ volume $>1.000 \mathrm{~m}^{3}$ ). Tempos de reverberação em torno de $0,8 \mathrm{~s}$ (nas bandas de oitava de 63 a $4.000 \mathrm{~Hz}$ ) foram obtidos pela simulação e medição de uma sala de trabalho em ambiente industrial, com volume de $455 \mathrm{~m}^{3}$ e coeficiente de absorção sonora médio de 0,12 (ALIABADI et al., 2014). Rindel (2012) e Rindel e Christensen (2012) obtiveram TR entre 0,3 e 1,1 s de três escritórios de plano aberto, pela medição e simulação conforme a ISO 3382-3 (ISO, 2012), nas bandas de oitava de 500 e $1.000 \mathrm{~Hz}$. O estudo de Zhang, Kang e Jiao (2012) sobre o impacto do ruído em quatro escritórios de plano aberto baseado em entrevistas e medições acústicas mostrou que o TR variou entre 0,4 e 1,2 s nas bandas de oitava entre 200 e $4.000 \mathrm{~Hz}$. Virjonen, Keränen e Hongisto (2009) apresentaram valores de TR para 16 escritórios de plano aberto que se mantiveram entre 0,32 a 1,15 s (bandas de oitava de 250 e $4.000 \mathrm{~Hz}$ ), cujas características de geometria da sala, mobiliário e absorção variaram consideravelmente.

No Brasil inexiste norma específica que determine tempos de reverberação recomendados para escritórios. Os ambientes para os quais a norma brasileira NBR 12179 (ABNT, 1992) recomenda tempos de reverberação ótimos são: estúdio de rádio ou para a palavra, sala de conferência e de concerto, cinema, estúdio de rádio para música, igrejas, entre outros. O TR é habitualmente aplicado como parâmetro no controle de ruído como um critério de projeto para interior de ambientes. No caso de estudos mais específicos e de dados de TR em escritórios instalados no interior de ambientes industriais, podem ser adotadas as recomendações de valores de TR de escritórios constantes nas normas VDI 2569 (VDI, 1990), ISO 11690-1 (ISO, 1996b) e AS/NZS 2107 (AS/NZS, 2016).

A inteligibilidade da fala é um descritor da qualidade sonora que pode ser utilizado para analisar a adequação de espaços onde a fala é essencial. Diversos estudos têm investigado a relação entre o Índice de Transmissão da Fala (STI), o ruído de fundo e o TR (MÜLLER, 2005; VENETJOKI et al., 2006; HAKA et al., 2009; HONGISTO et al., 2012; SOMMERHOFF; ROSAS, 2012; JAHNCKE; HONGISTO; VIRJONEN, 2013; VAN DE POLL et al., 2014). A classificação dos valores do STI define o (zero) como completamente ininteligível e 1 como ótima inteligibilidade, sendo que valores de 0,45 a 0,60 e de 0,60 a 0,75 correspondem às categorias razoável e bom, respectivamente (IEC, 2011). 
O STI é uma métrica física objetiva que quantifica a qualidade de transmissão da voz, em função da transferência de modulação e é avaliado pelo fator de redução de modulação (HOUTGAST; STEENEKEN, 1985; KITAPCl et al., 2013). Este fator depende de duas variáveis mensuráveis e presentes no meio de transmissão (ambiente), isto é, a relação sinal-ruído (LSN) que tem em conta o ruído de fundo e o TR. Os valores da LSN são estabelecidos para uma faixa de $\pm 15 \mathrm{~dB}$, sendo acima de $+15 \mathrm{~dB}$ considerado muito bom e abaixo de $-15 \mathrm{~dB}$, péssimo (HODGSON; NOSAL, 2002; MÜLLER, 2005). Galbrun e Kitapci (2014) mostraram que, para diferentes condições de TR, de LSN e independente da frequência, o STI foi constante (igual a zero) para valores inferiores a $-15 \mathrm{~dB}$ e também tendeu a ser independente da LSN para valores superiores $a+20 \mathrm{~dB}$, exceto para tempos de reverberação abaixo de 0,4 $\mathrm{s}$. Ainda, os pesquisadores confirmaram que as maiores variações do STI aconteciam para $-10 \mathrm{~dB} \leq \mathrm{LSN} \leq+10 \mathrm{~dB}$. Sommerhoff e Rosas (2012) observaram, no estudo de salas acusticamente diferentes que se caracterizam pela pobre inteligibilidade $(\mathrm{STI}<0,40)$, os valores da LSN seriam inferiores a $-3 \mathrm{~dB}$ com tempos de reverberação superiores a $1 \mathrm{~s}$. Na avaliação do conforto acústico de estações de trabalho em espaços abertos de bancos o STI foi de 0,56 $\pm 0,09$ e a LSN de 20,5 \pm 8.2 $\mathrm{dB}$, com tempos de reverberação em torno de 1,24 s (GOLMOHAMMADI; ALIABADI; NEZAMI, 2017). Os valores de STI entre 0,30 e 0,45 são considerados pobres para LSN entre +3 e +10 dB de acordo com a IEC 60268-16 (IEC, 2011).

Diversos estudos têm demonstrado a relevância da avaliação do STI em ambientes do tipo escritórios de plano aberto e a interferência do tempo de reverberação e do NPS do ruído de fundo na inteligibilidade da fala (PASSERO; ZANNIN, 2012a, 2012b; RINDEL; CHRISTENSEN, 2012; EBISSOU; PARIZET; CHEVRET, 2015; NOWOŚWIAT; OLECHOWSKA, 2016; CHEVRET, 2016; CHEVRET; PARIZET; KOSTALLARI, 2017). O trabalho de Chevret, Parizet e Kostallari (2017) apresentou uma nova métrica chamada de Irrelevant Sound Effect (ISE), baseada na medição da modulação temporal de curta duração dos NPS, cujos resultados indicam que esta métrica é tão eficiente quanto o STI para avaliação da inteligibilidade da fala com ruído de fundo. Uma estimativa rápida do STI usando uma função logarítmica e o tempo de reverberação da sala permitiu a Nowoświat e Olechowska (2016) verificarem um modelo computacional com diferentes volumes e formas. Dos seis ambientes reais avaliados e modelados, uma sala pequena (volume de $31,25 \mathrm{~m}^{3}$ e STI de 0,7 ) com paredes de chapa de compensado OSB foi utilizada como sala de teste em laboratório, três eram salas de leitura de uma universidade (volumes entre 300 e $600 \mathrm{~m}^{3}$, com STI entre $0,40-0,48$ ), outra sala era igreja (volume de $5.000 \mathrm{~m}^{3} \mathrm{e} \mathrm{STI}$ de 0,33 ) e uma casa de cultura (volume de $1.100 \mathrm{~m}^{3}$ e STI de 0,39). A pesquisa de Chevret (2016) demonstrou a influência do efeito da modulação do ruído ambiente na inteligibilidade da fala em termos da densidade de ocupação do local de trabalho para amostras sonoras reais e sintetizadas em laboratório. O potencial de perturbação do ambiente sonoro em um escritório de plano aberto foi avaliado por Ebissou, Parizet e Chevret (2015), para um STI entre 0,25 e 0,65, pela mensuração do desempenho e por entrevistas com 57 indivíduos para medir a carga cognitiva. Os resultados mostraram que os indivíduos com melhor desempenho relataram uma maior dificuldade em todas as condições ruidosas, embora seu desempenho não tenha diminuído. As avaliações de Passero e Zannin (2012a), baseadas em simulações acústicas, modificações da arquitetura e do ruído de fundo de escritórios de plano aberto, mostraram que boas condições de conforto acústico puderam ser alcançadas com painéis divisórios e teto com coeficiente de absorção sonora em torno de 0,8 . Em outro trabalho de Passero e Zannin (2012b) foi avaliada a variação do STI em um modelo computacional de um escritório de plano aberto a partir de mudanças arquitetônicas e do ruído de fundo de forma que a organização espacial dos grupos de trabalhos fornecesse uma razoável inteligibilidade da fala. 


\section{Avaliação por meio das curvas critério NC}

As curvas de critério de rú́do (NC) foram desenvolvidas por Beranek (1957) para estabelecer situações satisfatórias para a inteligibilidade da fala e convivência geral em ambientes. Um conjunto de curvas é fornecido juntamente com um número atribuído a cada uma (AYR et al., 2003). A curva NC é determinada pela curva mais baixa na qual nenhum ponto do espectro de frequência medido se encontre acima. São definidas em intervalos de $5 \mathrm{~dB}$ e, entre esses intervalos, são obtidas por interpolação. A Norma NBR 10152 (ABNT, 2017) apresenta uma tabela onde se encontram os valores de referência para diferentes usos de ambientes internos de uma edificação. $O$ ambiente é considerado adequado à sua finalidade quando os níveis de pressão sonora representativos forem iguais ou inferiores aos valores de referência. Os valores de referência para escritórios coletivos são os NPS da curva NC 40 e os valores aceitáveis para o uso do ambiente são os NPS da curva NC 45.

\section{Avaliação por meio das curvas critério de salas RC Mark II}

O método RC Mark II é derivado do método Room Criteria (RC) desenvolvido por Blazier jr (1981), porém se diferenciando no modo em que se determina um índice de qualidade baseado no espectro (BLAZIER JR, 1997; TOCCI, 2000).

A curva RC Mark II correspondente ao espectro medido é caracterizada pelo cálculo do PSIL. O resultado obtido é o valor da frequência de $1.000 \mathrm{~Hz}$ na curva RC Mark II. A classificação RC Mark II divide a faixa de frequências audíveis em três regiões: baixa (16 a $63 \mathrm{~Hz}$ ), média (125 a $500 \mathrm{~Hz}$ ) e alta $(1.000$ a $4.000 \mathrm{~Hz})$. O som que é ultrapassado nessas três faixas é caracterizado respectivamente como retumbante (rumble), rugido (roar) e assobiante (hiss) (BLAZIER JR, 1997; TOCCI, 2000). O Quality Assessment Index (QAI) é a diferença entre o maior e menor valor médio de energia do desvio espectral, representando o desequilíbrio do espectro em relação à neutralidade. Para calcular o nível médio de energia do desvio espectral nas três faixas de frequência são utilizadas as equações 1,2 e 3 (TOCCl, 2000):

$$
\begin{aligned}
& \Delta \mathrm{LF}=10 \log \left(\frac{10^{\frac{\Delta L_{16}}{10}}+10^{\frac{\Delta L_{31,5}}{10}}+10^{\frac{\Delta L_{63}}{10}}}{3}\right), \\
& \Delta \mathrm{MF}=10 \log \left(\frac{10^{\frac{\Delta L_{125}}{10}}+10^{\frac{\Delta L_{250}}{10}}+10^{\frac{\Delta L_{500}}{10}}}{3}\right), \\
& \Delta \mathrm{HF}=10 \log \left(\frac{10^{\frac{\Delta L_{1000}}{10}}+10^{\frac{\Delta L_{2000}}{10}}+10^{\frac{\Delta L_{4000}}{10}}}{3}\right) .
\end{aligned}
$$

Onde:

$\Delta \mathrm{LF}=$ Nível médio de energia do desvio espectral para baixa frequência; $\Delta \mathrm{MF}=$ Nível médio de energia do desvio espectral para média frequência; $\Delta \mathrm{HF}=$ Nível médio de energia do desvio espectral para alta frequência; $\Delta \mathrm{L}_{f}=$ Diferença entre o valor espectral e o valor correspondente da curva RC Mark II em determinada frequência.

Se o QAI for menor ou igual a $5 \mathrm{~dB}$, o espectro é considerado neutro, demonstrando um balanceamento adequado entre baixas, médias e altas frequências. Se o QAI for maior que $5 \mathrm{~dB}$, o espectro é qualificado de acordo com o maior nível médio de energia do desvio espectral, LF (baixa frequência), MF (média frequência) ou HF (alta frequência) e considerado desagradável. Caso o ruído seja maior que $65 \mathrm{~dB}$ na frequência de $16 \mathrm{~Hz}$ ou $31,5 \mathrm{~Hz}$ o espectro também será classificado como LFV $\mathrm{B}_{B}$ (que apresenta vibração 
moderadamente perceptível). Caso o ruído seja maior que $75 \mathrm{~dB}$ na frequência de $16 \mathrm{~Hz}$ ou $31,5 \mathrm{~Hz}$ o espectro é classificado como $\mathrm{LFV}_{\mathrm{A}}$ (que apresenta vibração claramente perceptível) (BLAZIER JR, 1997; TOCCl, 2000).

\section{Avaliação dos tempos de reverberação}

Sabine obteve a seguinte relação entre o tempo de reverberação e os parâmetros de uma sala (LONG, 2011):

$$
\begin{aligned}
& T R=0,164 \frac{V}{S \bar{\alpha}+4 m V}, \\
& \bar{\alpha}=\frac{\sum S_{i} \propto_{i}}{\sum S_{i}} .
\end{aligned}
$$

Onde:

$\mathrm{V}=$ Volume da sala $\left(\mathrm{m}^{3}\right)$;

$\mathrm{S}=$ Área de superfície da sala $\left(\mathrm{m}^{2}\right)$;

$\mathrm{S}_{\mathrm{i}}=$ Área da superfície i $\left(\mathrm{m}^{2}\right)$;

$\propto_{i}=$ Coeficiente de absorção sonora da superfície i;

$\bar{\alpha}=$ Coeficiente de absorção sonora equivalente;

$m=$ Coeficiente de absorção de energia da onda sonora pelo ar.

A equação de Sabine possui limitações. Sua equação é baseada na presunção que o campo acústico da sala é difuso. Assim as superfícies devem ser refletoras e os coeficientes de absorção das superfícies na ordem de 0,2 ou menos. Caso contrário, a fórmula de Sabine pode ser imprecisa (MEHTA; JOHNSON; ROCAFORT, 1999). Para ambientes com mais absorção Carl Eyring desenvolveu outra equação baseada em uma ideia atribuída a R. F. Norris. Em uma sala perfeitamente absorvente (câmara anecóica), a equação de Sabine dá um resultado que não é zero para o tempo de reverberação e, a partir as equações de Norris Eyring, a equação corretamente resulta em zero (LONG, 2011). A seguir são apresentadas as equações de Norris Eyring:

$$
\begin{aligned}
& T R=0,164 \frac{V}{-S \ln (1-\bar{\alpha})+4 m V}, \\
& \bar{\propto}=\frac{\sum S_{i} \propto_{i}+\sum A_{i}^{\prime}}{\sum S_{i}} .
\end{aligned}
$$

Onde:

$\mathrm{A}_{\mathrm{i}}^{\prime}=$ Absorção sonora de pessoas e objetos ( $\mathrm{m}^{2}$ Sabine).

$\mathrm{V}=$ Volume da sala $\left(\mathrm{m}^{3}\right)$;

$\mathrm{S}=$ Área de superfície da sala $\left(\mathrm{m}^{2}\right)$;

$\mathrm{S}_{\mathrm{i}}=$ Área da superfície i $\left(\mathrm{m}^{2}\right)$;

$\propto_{i}=$ Coeficiente de absorção sonora da superfície i;

$\bar{\alpha}=$ Coeficiente de absorção sonora equivalente;

$m=$ Coeficiente de absorção de energia da onda sonora pelo ar.

Para o tempo de reverberação de escritórios calculado mediante média aritmética entre as frequências de 500, 1.000 e $2.000 \mathrm{~Hz}$, a norma VDI 2569 (VDI, 1990) recomenda um valor menor ou igual a 0,5 S, a norma AS/NZS 2107 (AS/NZS, 2016) um valor menor ou igual a 0,6 s e a norma ISO 11690-1 (ISO, 1996b) recomenda para ambientes com até 200 $\mathrm{m}^{3}$ (salas pequenas) valores que estejam entre 0,5 e $0,8 \mathrm{~s}$ e, acima desse volume, entre 0,8 e $1,3 \mathrm{~s}$.

Avaliação do índice de transmissão da fala

O índice da transmissão da fala (STI) é calculado a partir do fator de redução de modulação $m\left(f_{m}\right)$, com $f_{m}$ sendo a frequência de modulação da fala e variando de 0,63 
a $12,5 \mathrm{~Hz}$ em bandas de $1 / 3$ de oitava e $m\left(f_{m}\right)$ calculado para bandas de frequência de oitava de $125 \mathrm{~Hz}$ a $8 \mathrm{kHz}$ (IEC, 2011). Para obter o STI, o nível do sinal-ruído aparente, $L_{S N a p p}$, expresso em (dB), deve ser calculado pela Equação 8 (HOUTGAST; STEENEKEN, 1985; LONG, 2011):

$$
L_{N S a p p}=10 \log \frac{m\left(f_{m}\right)}{1-m\left(f_{m}\right)} .
$$

Onde:

$L_{\text {NSapp }}=$ Nível do sinal-ruído aparente;

$m\left(f_{m}\right)=$ Fator de redução de modulação;

$f_{m}=$ Frequência de modulação da fala $(\mathrm{Hz})$.

O fator de redução de modulação pode ser calculado com base no tempo de reverberação (TR), em segundos, e no nível do sinal-ruído $\left(L_{N S}\right)$, em $\mathrm{dB}$, a partir da Equação 9 (HOUTGAST; STEENEKEN, 1985; LONG, 2011):

$$
\mathrm{m}\left(f_{m}\right)=\frac{1}{\sqrt{1+\left(2 \pi f_{m} \frac{\mathrm{TR}}{13,8}\right)^{2}}} \frac{1}{1+10^{-0,1 \mathrm{~L}_{\mathrm{SN}}}} .
$$

Onde:

TR = Tempo de reverberação (s);

$m\left(f_{m}\right)=$ Fator de redução de modulação;

$f_{m}=$ Frequência de modulação da fala $(\mathrm{Hz})$.

No cálculo do STI podem ser incluídos os fatores de ponderação específicos para homens e mulheres, a e $\beta$, respectivamente, para cada banda de frequência de oitava, que são definidas pela língua inglesa pela norma IEC 60268-16 (IEC, 2011) e neste trabalho foram utilizadas para o sexo masculino do locutor.

$$
\mathrm{STI}=\sum_{\mathrm{n}=1}^{7} \alpha_{\mathrm{n}} \mathrm{L}_{\mathrm{SNapp}_{\mathrm{n}}}-\sum_{\mathrm{n}=1}^{6} \beta_{\mathrm{n}} \sqrt{\mathrm{L}_{\mathrm{SNapp}_{\mathrm{n}}} \mathrm{L}_{\mathrm{SNapp}_{\mathrm{n}+1}}} .
$$

Onde:

$L_{\text {NSapp }}=$ Nível do sinal-ruído aparente;

$\alpha, \beta=$ Fatores de ponderação.

\section{Método}

Três escritórios - Escritório 1, Escritório 2 e Escritório 3 (E1, E2 e E3) - localizados na casa de força de uma usina hidrelétrica foram avaliados quanto à interferência do ruído de fundo e do TR na inteligibilidade da fala, a partir da quantificação e análise dos critérios - Noise Criterion (NC) e Room Criterion (RC Mark II), e do Índice de Transmissão da Fala (STI). O ruído nos escritórios era ininterrupto devido à contínua operação da usina para a produção de energia elétrica.

\section{Caracterização dos escritórios}

Os espaços construídos analisados são utilizados para trabalho relacionado com manutenção e operação da usina, como consulta de projetos, reuniões, etc. O Ambiente 3 (A3) é um depósito, no entanto foi avaliado como escritório, para futura transformação.

No Tabela 1 são apresentadas as características dos escritórios E1, E2 e E3, como volume interno, área interna e altura livre entre piso e teto (pé-direito). No Quadro 1 são apresentados os materiais de acabamento das superfícies internas dos escritórios e tipo 
de ar condicionado. Na Figura 1 são apresentadas informações complementares de sistemas construtivos utilizados, tipos e tamanhos de aberturas.

Tabela 1 - Características dimensionais dos ambientes dos escritórios avaliados.

\begin{tabular}{|c|c|c|c|c|}
\hline Esc & Ambiente & Volume interno $\left[\mathrm{m}^{3}\right]$ & Área interna $\left[\mathrm{m}^{2}\right]$ & Altura livre [m] \\
\hline \multirow{3}{*}{ E1 } & A1 & 70,8 & 27,1 & \multirow{3}{*}{2,61} \\
\hline & $\mathrm{A} 2$ & 36,5 & 14 & \\
\hline & A3 & 70,8 & 27,1 & \\
\hline \multirow{2}{*}{ E2 } & A4 & 169,6 & 65,2 & \multirow{2}{*}{2,60} \\
\hline & A5 & 95,6 & 36,8 & \\
\hline E3 & A6 & 1062,6 & 339,5 & 3,13 \\
\hline
\end{tabular}

Fonte: Os autores

Quadro 1 - Materiais de acabamento das superfícies internas dos ambientes dos escritórios avaliados e tipo de ar condicionado.

\begin{tabular}{|c|c|c|c|c|}
\hline \multicolumn{2}{|c|}{ Escritório / Ambiente } & Teto & Paredes & Ar condicionado \\
\hline \multirow{3}{*}{ E1 } & $\mathrm{A} 1$ & \multirow{5}{*}{ Forro fibra mineral } & \multirow{6}{*}{ Reboco e pintura } & \multirow{5}{*}{ Split } \\
\hline & $\mathrm{A} 2$ & & & \\
\hline & A3 & & & \\
\hline \multirow{2}{*}{ E2 } & A4 & & & \\
\hline & A5 & & & \\
\hline E3 & A6 & Forro vermiculita & & Self Contained \\
\hline
\end{tabular}

Fonte: Os autores

Os móveis eram predominantemente fabricados em MDF revestidos com laminado melamínico e as cadeiras estofadas. No Ambiente 3 ( $\left.\mathrm{A}_{3}\right)$, os armários possuíam estrutura metálica e prateleiras em madeira compensada.

O Tabela 2 apresenta os coeficientes de absorção ( $\alpha$ ), em função da frequência, dos materiais de acabamento das superfícies dos escritórios estudados.

Os três escritórios foram instalados sobre o piso interno da casa de força da usina utilizando-se de sistema de piso flutuante com acabamento vinílico.

Tabela 2 - Coeficientes de absorção sonora $(\alpha)$ dos materiais de acabamento das superfícies dos escritórios avaliados.

\begin{tabular}{|c|c|c|c|c|c|c|}
\hline \multirow{2}{*}{ Materiais } & \multicolumn{6}{|c|}{ Frequência (Hz) } \\
\hline & 125 & 250 & 500 & 1.000 & 2.000 & 4.000 \\
\hline Cadeiras $^{1}$ & 0,19 & 0,37 & 0,56 & 0,67 & 0,61 & 0,59 \\
\hline Forro fibra mineral ${ }^{3}$ & 0,05 & 0,26 & 0,67 & 0,87 & 0,97 & 0,99 \\
\hline Forro vermiculita $^{3}$ & 0,68 & 0,76 & 0,6 & 0,65 & 0,82 & 0,76 \\
\hline Divisórias e Móveis ${ }^{3}$ & 0,03 & 0,03 & 0,04 & 0,04 & 0,04 & 0,04 \\
\hline Piso vinílico ${ }^{1}$ & 0,02 & 0,03 & 0,03 & 0,03 & 0,03 & 0,02 \\
\hline Porta de madeira ${ }^{2}$ & 0,15 & 0,11 & 0,1 & 0,07 & 0,06 & 0,07 \\
\hline Reboco $^{2}$ & 0,03 & 0,03 & 0,04 & 0,04 & 0,04 & 0,04 \\
\hline Vazios $^{2}$ & 1 & 1 & 1 & 1 & 1 & 1 \\
\hline Vidro $^{2}$ & 0,35 & 0,25 & 0,18 & 0,12 & 0,07 & 0,04 \\
\hline
\end{tabular}

Fonte: Long $(2011)^{1}$, Bistafa $(2011)^{2}$ e catálogos técnicos ${ }^{3}$ dos materiais, elaborado pelos autores 
Figura 1 - Plantas dos escritórios avaliados, com indicação dos pontos de medição de NPS Escritório 1 (E1)

(11) (I1)

P1
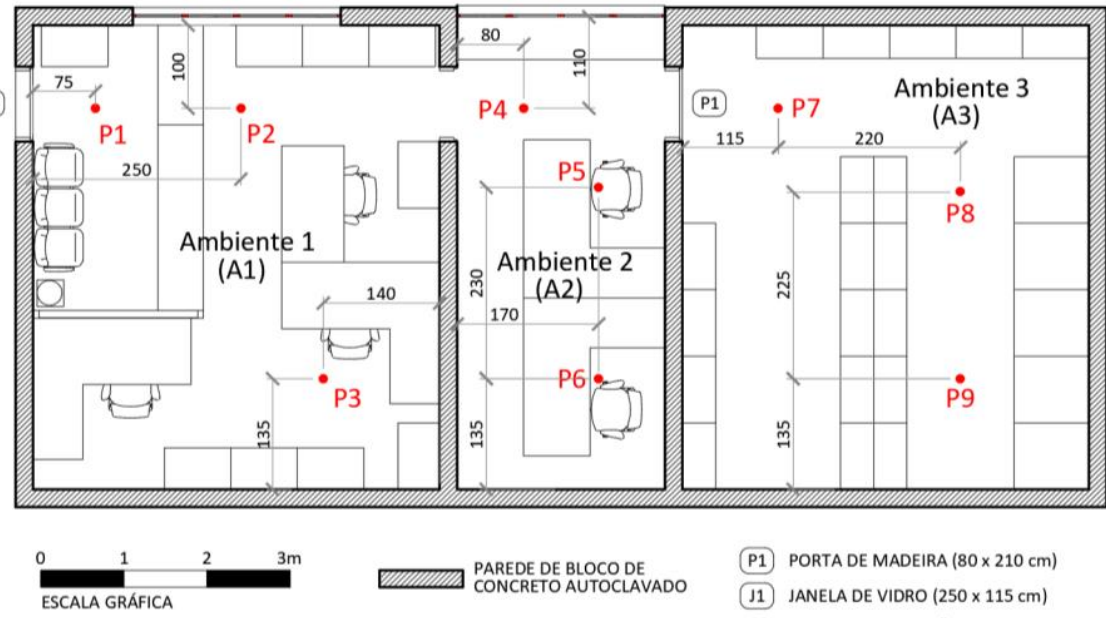

P1) PORTA DE MADEIRA $(80 \times 210 \mathrm{~cm})$

J1) JANELA DE VIDRO $(250 \times 115 \mathrm{~cm})$

Pi PONTOS DE MEDIÇÃO

Escritório 2 (E2)

J2

J2

P2
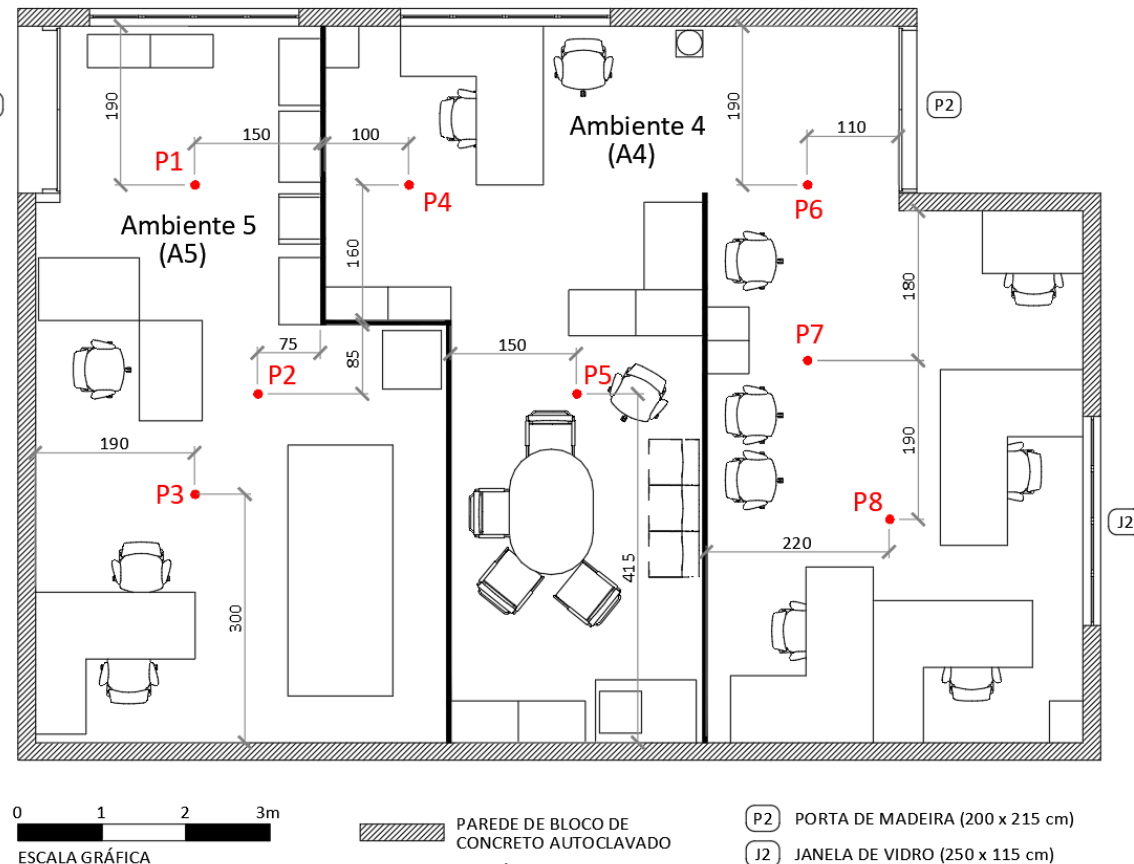

TIIIIII
PAREDE DE BLOCO DE
CONCRETO AUTOCLAVAD

P2 PORTA DE MADEIRA $(200 \times 215 \mathrm{~cm})$ ESCALA GRÁFICA

— DIVISÓRIA NAVAL

JANELA DE VIDRO $(250 \times 115 \mathrm{~cm})$

Pi PONTOS DE MEDIÇÃO

Escritório 3 (E3)

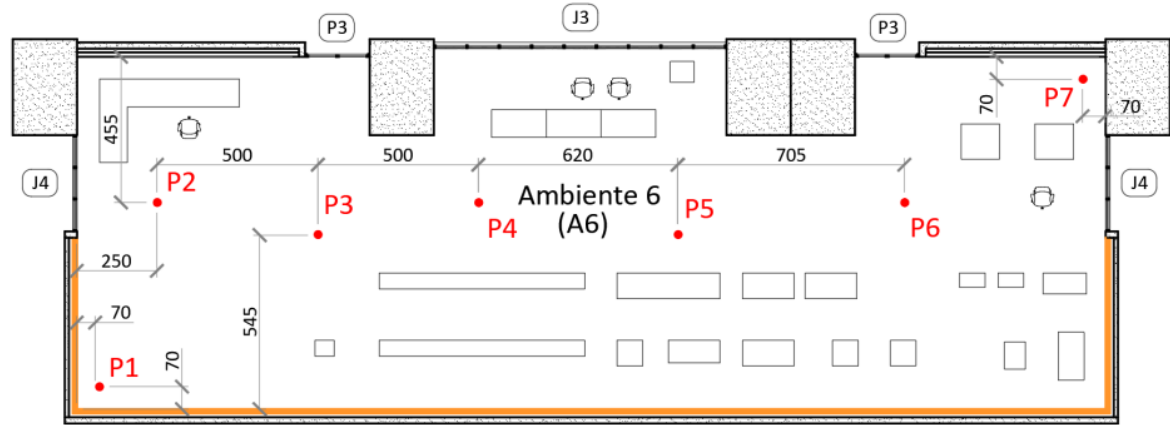

$$
\begin{array}{lll}
0 \text { ESCALA GRÁFICA } & 5 \mathrm{~m} \text { CONCRETO MACIÇO } & \text { P3 PORTA DE VIDRO }(200 \times 300 \mathrm{~cm}) \\
\text { PAREDE DE TIJOLO MACIÇO } & \text { J3 JANELA DE VIDRO }(910 \times 280 \mathrm{~cm}) \\
& \text { J4 JANELA DE VIDRO }(300 \times 280 \mathrm{~cm}) \\
& \text { Pi PONTOS DE MEDIÇÃO }
\end{array}
$$

Fonte: Os autores. 


\section{Avaliação acústica dos escritórios}

Para avaliar as condições acústicas dos ambientes foram utilizadas medições acústicas em campo, em pontos demarcados em todas as salas, seguindo as recomendações da norma NBR 10152 (ABNT, 2017). Para executar as medições utilizou-se um conjunto da fabricante $01 \mathrm{~dB}$ composto de um sonômetro Fusion integrador classe 1, um calibrador acústico Cal $21(1.000 \mathrm{~Hz}$ e $94 \mathrm{~dB}$, ref.: 0,00002 Pa) e o programa computacional dBTrait para aquisição e processamento dos dados. Os níveis foram medidos nas bandas de frequência de $1 / 3$ de oitava, de 16 a $10.000 \mathrm{~Hz}$. Um tripé posicionado a 1,3 m do solo foi utilizado para fixação do equipamento de medição. Foi utilizada a integração temporal $\mathrm{S}$ (slow) e a medição dos NPS em decibels (dB) (ABNT, 2017). Cada medição teve duração de 1 minuto e foi realizada três vezes, sendo obtido o $L_{\text {eq }}$ por bandas de frequência e global a partir da média das três medições. As posições do sonômetro utilizadas podem ser vistas na Figura 1. O tempo escolhido de 1 minuto para as medições deve-se ao fato de o ruído no local ser contínuo. Este tempo engloba todos os possíveis acontecimentos ruidosos, conforme recomendação da norma NBR 10152 (ABNT, 2017).

As unidades geradoras estavam em funcionamento durante todas as medições. Os materiais e equipamentos encontrados no interior das salas não foram retirados. Portas e janelas existentes foram fechadas. Os ambientes foram desocupados, estando presente nos locais das medições somente o operador do sonômetro.

Realizaram-se duas rodadas de medições. A primeira, com os equipamentos eletrônicos e ar condicionado ligados e a segunda, com os mesmos desligados. Não houve diferença nos resultados das medições de NPS em ambas as situações, constatando-se que o ruído desses equipamentos poderia ser desprezado do ruído de fundo.

Com os resultados de níveis de pressão sonora medidos foi possível avaliar o conforto acústico mediante as curvas NC (BERANEK, 1957; ABNT, 2017) e RC Mark II (BLAZIER JR, 1997).

Para avaliação por meio das curvas NC, consideraram-se, para níveis de conforto acústico apropriados para escritórios coletivos, os NPS da curva NC 40 estabelecida pela norma NBR 10152 (ABNT, 2017). O espectro utilizado para avaliação do ruído de fundo por meio das curvas NC foi o de bandas de frequência de oitava de 16 a $8.000 \mathrm{~Hz}$, e foram utilizados os NPS de cada banda de frequência medida nos pontos demarcados no interior de cada ambiente (Figura 1).

Para avaliação por meio do método RC Mark II, o PSIL foi calculado utilizando-se a média aritmética entre os níveis de pressão sonora das bandas de frequência de oitava de 500, 1.000 e $2.000 \mathrm{~Hz}$. Posteriormente gerou-se a curva RC Mark II. Com a diferença aritmética entre os valores do espectro medido e a curva RC Mark II em cada frequência, foram calculados os níveis médios de energia do desvio espectral utilizando-se as equações 1, 2 e 3. A partir dos níveis médios de energia do desvio espectral obteve-se o QAI. Com os resultados as salas foram classificadas, avaliando-se se também o balanceamento do espectro e se as baixas frequências (16 e $31,5 \mathrm{~Hz}$ ) produziam nos ocupantes a sensação de vibração no ambiente.

Os tempos de reverberação foram calculados nas bandas de frequência de oitava de 125 a $4.000 \mathrm{~Hz}$ e verificou-se o atendimento às normas internacionais. Para medição in loco do tempo de reverberação seria preciso gerar um NPS $45 \mathrm{~dB}$ acima do NPS máximo medido em cada banda de frequência de interesse, conforme norma ISO 3382-2 (ISO, 2008). Como o NPS no interior das salas estudadas era elevado e não era possível que a fonte de ruído fosse desligada, a determinação do tempo de reverberação a partir de 
medições tornou-se inviável. Para os cálculos foram utilizadas as equações de Sabine e Eyring (Equações 4 e 6).

O STI foi calculado pelas Equações 8, 9 e 10 a partir dos dados de tempo de reverberação de cada ambiente em cada escritório e para os valores da relação sinal-ruído ( $\left.L_{S N}\right)$ de -5 , 0 e $+5 \mathrm{~dB}$, nas bandas de frequência de oitava entre 125 e $8.000 \mathrm{~Hz}$.

\section{Resultados e discussão}

A Tabela 3 mostra o resultado das medições dos NPS de todos os ambientes dos escritórios avaliados. A Tabela 4 apresenta os valores calculados de TR com aplicação das equações de Sabine e Eyring. Os NPS representativos dos ambientes foram obtidos por meio de médias logarítmicas realizadas entre todos NPS medidos no interior de cada ambiente. A Figura 2 mostra o espectro medido no interior do Escritório 1 (E1) comparado com as curvas NC. Observa-se que em diversas bandas de frequência o NPS encontrado ultrapassou o limite estabelecido para conforto (curva NC 40), sendo a faixa que varia de 125 a $500 \mathrm{~Hz}$ a mais crítica. Portanto nenhum dos ambientes do Escritório 1 (E1) estava de acordo com a curva estipulada para conforto acústico. Somente o Ambiente 3 atendeu à curva NC 45, aceitável para conforto.

Com os NPS medidos (Tabela 3) foi possível avaliar o conforto acústico mediante as curvas NC (BERANEK, 1957; ABNT, 2017) e RC Mark II (BLAZIER JR, 1997). Com os TR calculados pôde-se avaliar o atendimento às normas internacionais e também calcular $O$ STI dos ambientes. Todos os resultados obtidos são apresentados na Tabela 5, que fornece um resumo da situação dos ambientes avaliados.

Tabela 3 - NPS dos ambientes dos escritórios analisados

\begin{tabular}{|c|c|c|c|c|c|c|c|c|c|c|c|}
\hline \multicolumn{12}{|c|}{ Leq (dB) } \\
\hline Escritório & Ambiente & $16 \mathrm{~Hz}$ & $31,5 \mathrm{~Hz}$ & $63 \mathrm{~Hz}$ & $125 \mathrm{~Hz}$ & $250 \mathrm{~Hz}$ & $500 \mathrm{~Hz}$ & $1 \mathrm{kHz}$ & $2 \mathrm{kHz}$ & $4 \mathrm{kHz}$ & $8 \mathrm{kHz}$ \\
\hline \multirow{3}{*}{ E1 } & A1 & 74 & 75 & 62 & 58 & 68 & 52 & 40 & 31 & 19 & 13 \\
\hline & $A 2$ & 81 & 76 & 60 & 60 & 68 & 53 & 41 & 30 & 21 & 14 \\
\hline & A3 & 70 & 75 & 59 & 57 & 63 & 46 & 35 & 25 & 18 & 13 \\
\hline \multirow{2}{*}{ E2 } & A4 & 75 & 67 & 63 & 65 & 67 & 54 & 44 & 36 & 27 & 18 \\
\hline & A5 & 76 & 63 & 62 & 62 & 67 & 55 & 45 & 38 & 28 & 23 \\
\hline E3 & A6 & 77 & 74 & 67 & 68 & 74 & 62 & 58 & 56 & 40 & 27 \\
\hline
\end{tabular}

Fonte: Os autores.

Tabela 4 - TR de Sabine e Eyring dos ambientes dos escritórios analisados

\begin{tabular}{cccccccc}
\hline \multicolumn{7}{c}{ TR Sabine (s) / TR Eyring (s) } \\
\hline Escritório & Ambiente & $\mathbf{1 2 5} \mathbf{~ H z}$ & $\mathbf{2 5 0} \mathbf{~ H z}$ & $\mathbf{5 0 0} \mathbf{~ H z}$ & $\mathbf{1 ~} \mathbf{~ H z}$ & $\mathbf{2 ~} \mathbf{~ H z}$ & $\mathbf{4 ~ k H z}$ \\
\hline \multirow{2}{*}{ E1 } & A1 & $1,6 / 1,5$ & $0,9 / 0,8$ & $0,5 / 0,4$ & $0,4 / 0,3$ & $0,4 / 0,3$ & $0,3 / 0,3$ \\
\cline { 3 - 8 } & A2 & $1,1 / 1,0$ & $0,7 / 0,7$ & $0,4 / 0,4$ & $0,3 / 0,3$ & $0,3 / 0,3$ & $0,3 / 0,3$ \\
\cline { 3 - 8 } & A3 & $3,2 / 3,1$ & $1,8 / 1,7$ & $0,9 / 0,8$ & $0,8 / 0,7$ & $0,7 / 0,6$ & $0,7 / 0,6$ \\
\hline \multirow{2}{*}{ E2 } & A4 & $2,5 / 2,4$ & $1,1 / 1,0$ & $0,5 / 0,4$ & $0,4 / 0,3$ & $0,4 / 0,3$ & $0,4 / 0,3$ \\
\hline & A5 & $2,4 / 2,3$ & $1,1 / 1,0$ & $0,5 / 0,5$ & $0,4 / 0,4$ & $0,4 / 0,3$ & $0,4 / 0,3$ \\
\hline E3 & A6 & $0,6 / 0,6$ & $0,6 / 0,5$ & $0,7 / 0,6$ & $0,7 / 0,6$ & $0,6 / 0,5$ & $0,5 / 0,5$ \\
\hline
\end{tabular}

Fonte: Os autores. 
MARTINS, Rodrigo Valdeci; MIRANDA, Erasmo Felipe Vergara

Conforto acústico de escritórios em ambiente industrial de usina hidrelétrica

\begin{tabular}{|c|c|c|c|c|c|c|c|}
\hline \multicolumn{2}{|c|}{$\begin{array}{l}\text { Índice / Critério de } \\
\text { avaliação acústica }\end{array}$} & \multicolumn{3}{|c|}{ E1 } & \multicolumn{2}{|c|}{ E2 } & \multirow{2}{*}{$\begin{array}{l}\text { E3 } \\
\mathrm{A6} \\
\end{array}$} \\
\hline & & A1 & A2 & A3 & A4 & A5 & \\
\hline \multicolumn{2}{|c|}{ Curva NC } & 48 & 52 & 43 & 61 & 61 & 69 \\
\hline \multicolumn{2}{|c|}{ PSIL } & 41 & 41 & 35 & 45 & 46 & 59 \\
\hline \multicolumn{2}{|c|}{ RC Mark II } & 41 (MF) & 41 (MF) & 35 (MF) & 45 (MF) & 46 (MF) & 59 (MF) \\
\hline \multicolumn{2}{|c|}{ QAI } & 16,8 & 16,3 & 16,8 & 12,0 & 10,5 & 10,5 \\
\hline \multicolumn{2}{|c|}{ LFVA / LFVB } & LFVA & LFVA & LFVA & LFVA & LFVA & LFVA \\
\hline \multirow{3}{*}{$\begin{array}{c}\text { TR } \\
\text { Sabine } \\
\text { (s) }\end{array}$} & VDI 2569 & $0,3^{1}$ & $0,3^{1}$ & 0,8 & $0,4^{1}$ & $0,4^{1}$ & 0,7 \\
\hline & $\begin{array}{c}A S / N Z S \\
2107\end{array}$ & $0,3^{1}$ & $0,3^{1}$ & 0,8 & $0,4^{1}$ & $0,4^{1}$ & 0,7 \\
\hline & ISO 11690-1 & $0,3^{1}$ & $0,4^{1}$ & $0,8^{1}$ & $0,5^{1}$ & $0,5^{1}$ & $0,6^{1}$ \\
\hline \multirow{3}{*}{$\begin{array}{c}\text { TR } \\
\text { Eyring } \\
\text { (s) }\end{array}$} & VDI 2569 & $0,3^{1}$ & $0,3^{1}$ & 0,7 & $0,3^{1}$ & $0,4^{1}$ & 0,6 \\
\hline & $\begin{array}{c}\text { AS/NZS } \\
2107\end{array}$ & $0,3^{1}$ & $0,3^{1}$ & 0,7 & $0,3^{1}$ & $0,4^{1}$ & $0,6^{1}$ \\
\hline & ISO 11690-1 & $0,3^{1}$ & $0,3^{1}$ & $0,8^{1}$ & $0,4^{1}$ & $0,4^{1}$ & $0,5^{1}$ \\
\hline \multirow{3}{*}{ STI } & -5 & 0,26 & 0,26 & 0,22 & 0,26 & 0,26 & 0,24 \\
\hline & 0 & 0,40 & 0,40 & 0,35 & 0,40 & 0,39 & 0,38 \\
\hline & 5 & 0,52 & 0,52 & 0,45 & 0,52 & 0,51 & 0,49 \\
\hline \multicolumn{2}{|c|}{ Classificação STI } & $\begin{array}{c}\text { Ruim } \\
\text { Pobre } \\
\text { Razoável }\end{array}$ & $\begin{array}{c}\text { Ruim } \\
\text { Pobre } \\
\text { Razoável }\end{array}$ & $\begin{array}{l}\text { Ruim } \\
\text { Pobre }\end{array}$ & $\begin{array}{c}\text { Ruim } \\
\text { Pobre } \\
\text { Razoável }\end{array}$ & $\begin{array}{c}\text { Ruim } \\
\text { Pobre } \\
\text { Razoável }\end{array}$ & $\begin{array}{c}\text { Ruim } \\
\text { Pobre } \\
\text { Razoável }\end{array}$ \\
\hline
\end{tabular}

1 : Valores que atendem as normas. Fonte: Os autores.

No Escritório 2 (Figura 3), os NPS encontrados ultrapassaram o limite estabelecido para conforto (curva NC 40) em diversas bandas de frequência, de $125 \mathrm{a} 1.000 \mathrm{~Hz}$, tanto no Ambiente 4 (A4) quanto no Ambiente 5 (A5), não atendendo os NPS estipulados para conforto acústico. No Escritório 3 (Figura 4) os NPS encontrados ultrapassaram o limite estabelecido para conforto (curva NC 40) em diversas bandas de frequência, de 63 a $4.000 \mathrm{~Hz}$, não atendendo aos estipulados para conforto acústico.

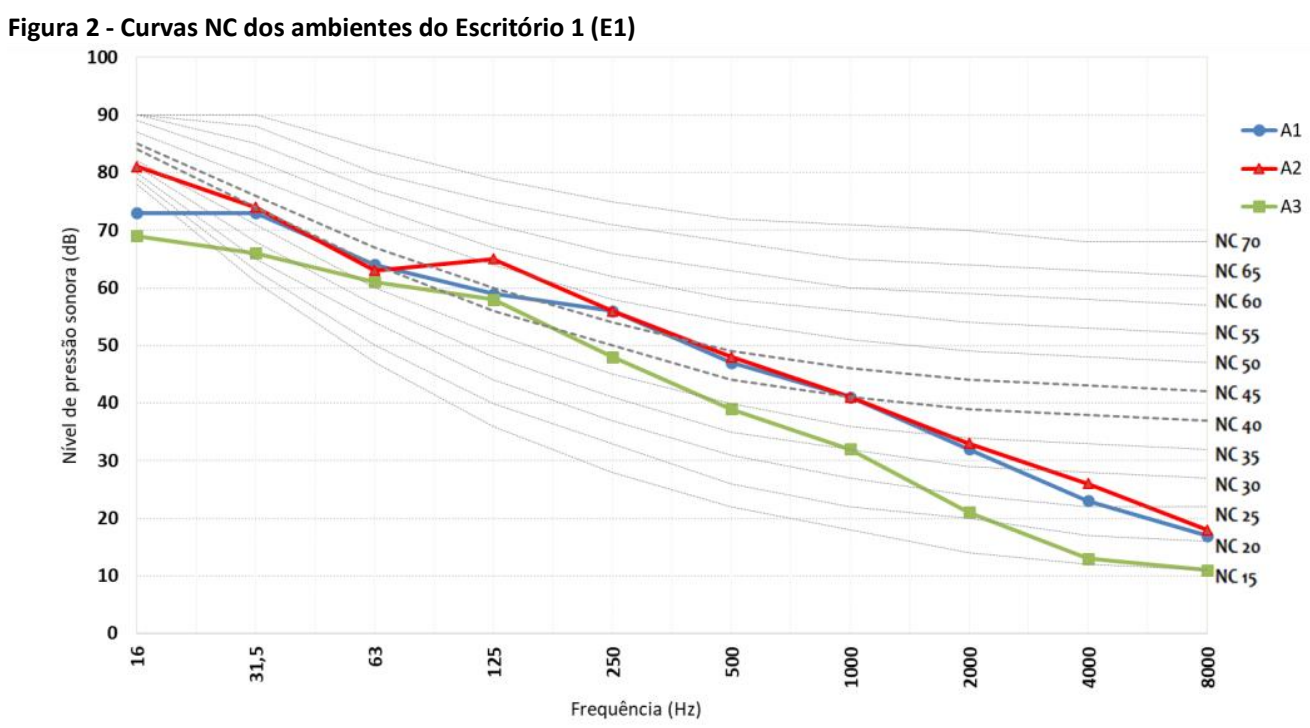

Fonte: Os autores. 


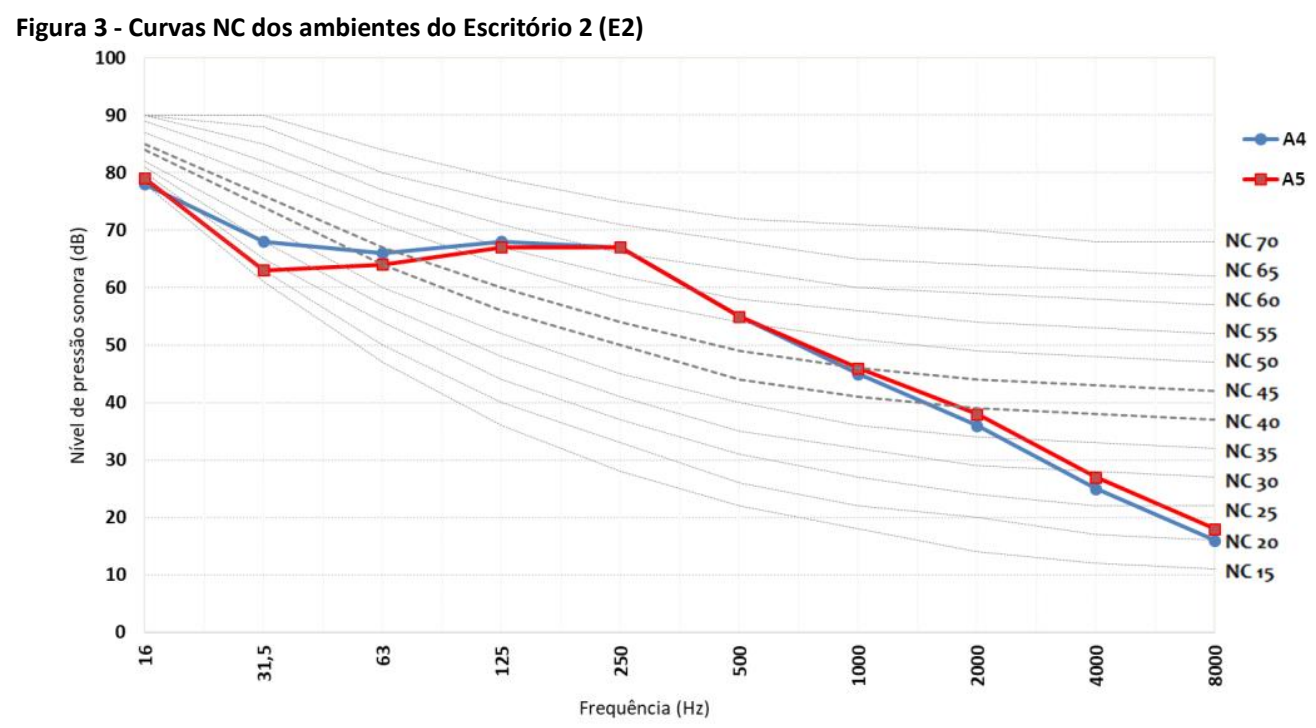

Fonte: Os autores.

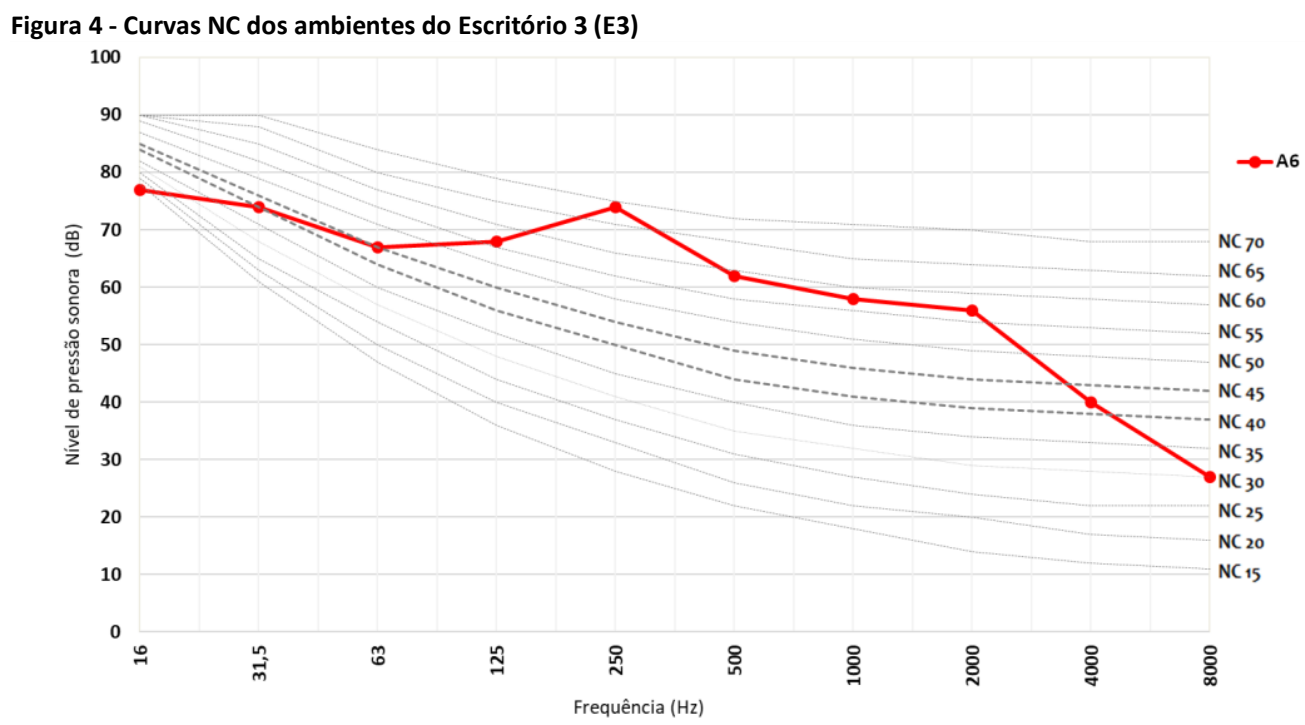

Fonte: Os autores.

As características do ambiente ruidoso e a falta de projeto acústico específico para os escritórios avaliados contribuíram para os altos valores de NPS encontrados. Um projeto acústico poderia reduzir o ruído de fundo nos escritórios, aumentando o conforto dos usuários dos espaços.

Os espectros sonoros de todos os ambientes dos escritórios avaliados foram qualificados como desbalanceados na faixa de média frequência predominante (125 a $500 \mathrm{~Hz}$ ) e caracterizados como rugido (roar), de acordo com a avaliação RC Mark II. Os ambientes do Escritório 1 (E1) apresentaram os menores valores de PSIL (35 a $41 \mathrm{~dB}$ ). Os valores de QAI encontrados superaram os $10 \mathrm{~dB}$, classificando o espectro de todos os ambientes avaliados como desagradável. Os valores de NPS medidos nas bandas de frequência de 16 ou $31,5 \mathrm{~Hz}$ apresentaram-se iguais ou acima de $75 \mathrm{~dB}$, classificando-os como $\mathrm{LFV}_{\mathrm{A}}$, isto é, com vibração claramente perceptível pelos ocupantes dos espaços.

A maioria dos ambientes avaliados atenderam aos tempos de reverberação recomendados pelas normas internacionais (Tabela 5). Nos ambientes com valores acima do recomendado, um maior conforto acústico poderia ser alcançado com a redução do TR, utilizando-se materiais de acabamento absorventes acústicos (AL HORR 
et al., 2016; REINTEN et al., 2017) como, por exemplo, a utilização de um forro com coeficiente de absorção sonora maior que 0,8 (PASSERO E ZANNIN, 2012a). Aumentando a absorção sonora dos materiais de acabamento das salas reduziria também o ruído de fundo, levando ao aumento da inteligibilidade da fala (REINTEN et al., 2017), pois todos os ambientes avaliados apresentaram STI classificados entre ruim e razoável.

\section{Conclusões}

Esta pesquisa investigou o conforto acústico de seis ambientes de três escritórios situados em ambiente industrial de usina hidrelétrica. Os resultados das medições acústicas mostraram valores de NPS superiores aos da curva critério de ruído NC 40, não atendendo à condição mínima de conforto acústico sugerida pela norma brasileira NBR 10152 (ABNT, 2017). Os valores de NPS que mais excederam os limites normativos, em todos os ambientes, foram os das bandas de frequência de 125 a $500 \mathrm{~Hz}$, o que aponta para características do ruído da casa de força da usina e direciona para uma correção por meio de isolamento das vedações externas dos escritórios, sendo recomendada uma avaliação da perda de transmissão sonora destas.

O espectro desbalanceado na faixa de média frequência (125 a $500 \mathrm{~Hz}$ ), evidenciado pela aplicação do método RC Mark II, confirma o resultado obtido com a avaliação das curvas NC. Os valores de QAl em todos os escritórios superaram os $10 \mathrm{~dB}$, classificando-os como desagradáveis. Em todos os ambientes as medições de NPS nas bandas de frequência de 16 ou $31,5 \mathrm{~Hz}$ apresentaram valores iguais ou superiores a $75 \mathrm{~dB}$ (classificados em $\mathrm{LFV}_{\mathrm{A}}$ ), havendo a percepção de vibração pelos ocupantes do espaço segundo método RC Mark II. Recomenda-se, portanto, uma avaliação mais aprofundada sobre conforto vibratório nos escritórios.

A maioria dos ambientes estudados atendeu aos tempos de reverberação estabelecidos para escritórios (variando entre 0,3 e 0,5 se). O Ambiente 3 ( $A 3$ ) e o Ambiente 6 (A6) necessitam modificações na sua arquitetura para que atendam aos TR recomendados: aumento de absorção de materiais de revestimento aplicados.

O índice de transmissão da fala nos três escritórios foi classificado de ruim a razoável, o que aponta para a necessidade da melhora do grau de inteligibilidade da fala, mediante redução do NPS e TR.

As medições e avaliações acústicas neste estudo revelaram que os aspectos acústicos do ruído e conforto acústico não foram totalmente considerados na fase de projeto dos escritórios avaliados. É uma situação comum, pois poucos países possuem diretrizes publicadas para elaboração deste tipo de projeto. A casa de força da usina hidrelétrica estudada não favorece a instalação de escritórios que não sejam devidamente projetados para utilização prolongada em trabalhos intelectuais e sem interferência no conforto e na comunicação oral.

\section{Notas}

(1) Casa de força: parte de uma usina hidrelétrica que concentra os equipamentos eletromecânicos responsáveis pela produção de energia (a turbina, o gerador e demais sistemas auxiliares). A velocidade de rotação de cada turbina está em torno de 90 RPM.

\section{Referências}

AL HORR, Y. et al. Occupant productivity and office indoor environment quality: A review of the literature. Building and Environment, v. 105, p. 369-389, 2016. DOI: https://doi.org/10.1016/j.buildenv.2016.06.001 
ALIABADI, M. et al. Development of an empirical acoustic model for predicting reverberation time in typical industrial workrooms using artificial neural networks. Acta Acustica united with Acustica, v. 100, p. 1090-1097, 2014. DOI: https://doi.org/10.3813/AAA.918788

ABNT - ASSOCIAÇÃO BRASILEIRA DE NORMAS TÉCNICAS. NBR 10152: Níveis de pressão sonora em ambientes internos a edificações. Brasil, 2017.

ABNT - ASSOCIAÇÃO BRASILEIRA DE NORMAS TÉCNICAS. NBR 12179: Tratamento acústico em recintos fechados. Brasil, 1992.

ANSI/ASA - AMERICAN NATIONAL STANDARDS INSTITUTE / ACOUSTICAL SOCIETY OF AMERICA. ANSI/ASA S3.14. American National Standard for Rating Noise with respect to speech interference. United States of America, 1977.

AS/NZS - AUSTRALIAN / NEW ZEALAND STANDARD. AS/NZS 2107: Acoustics - Recommended design sound levels and reverberation times for building interiors. Australia / New Zealand, 2016.

ASHRAE - AMERICAN SOCIETY OF HEATING, REFRIGERATING AND AIR-CONDITIONING ENGINEERS. Sound and Vibration. In: ASHRAE. Fundamentals Handbook. Atlanta: American Society of HVAC Engineers, Inc, 2001. cap. 7, p. 7.1-7.18.

AYR, U. et al. A new approach to assessing the performance of noise indices in buildings. Applied Acoustics, v. 64 , n. 2, p. 129-145, 2003. DOI: https://doi.org/10.1016/S0003-682X(02)00075-0

AYR, U.; CIRILLO, E; MARTELLOTTA, F. An experimental study on noise indices in air conditioned offices. Applied Acoustics, v. 62, p. 633-643, 2001. DOI: https://doi.org/10.1016/S0003-682X(00)00072-4

AYR, U.; CIRILLO, E; MARTELLOTTA, F. Further investigations of a new parameter to assess noise annoyance in airconditioned buildings. Energy and Buildings, v. 34, p. 765-774, 2002. DOI: https://doi.org/10.1016/S0378-

7788(02)00095-6

BERANEK, L. L. Revised criteria for noise in buildings. Noise Control, v. 3, n. 19, 1957. DOI: https://doi.org/10.1121/1.2369239

BLAZIER JR, W. E. Revised noise criteria for application in the acoustical design and rating of HVAC systems. Noise Control Engineering Journal, v.16, n. 2, p. 64-73, march-april, 1981. DOI: https://doi.org/10.3397/1.2832172

BLAZIER JR, W. E. Sound quality consideration in rating noise from heating, ventilating and air conditioning (HVAC) systems in buildings. Noise Control Engineering Journal, v. 43, n. 3, p. 53-63, mai. 1995. DOI: https://doi.org/10.3397/1.2828365

BLAZIER JR, W. E. RC Mark II: A refined procedure for rating the noise of heating, ventilating, and air-conditioning (HVAC) systems in buildings. Noise Control Engineering Journal, v. 45, N. 6, p. 243-250, 1997. DOI: https://doi.org/10.3397/1.2828446

BOWDEN, E. E.; WANG, L. M. Appropriate Characterization of Background Noise Levels in the Workplace. Architectural Engineering -- Faculty Publications, 11, 2006.

CHEVRET, P. Release from masking of speech intelligibility due to fluctuating ambient noise in open-plan offices. Applied Acoustics. v. 101, p. 156-167, 2016. DOI https://doi.org/10.1016/j.apacoust.2015.08.010

CHEVRET, P.; PARIZET, E.; KOSTALLARI, K. A simple sound metric for evaluating sound annoyance in open-plan offices. In: INTERNATIONAL CONGRESS ON NOISE AS A PUBLIC HEALTH PROBLEM, 12., 2017, Zurick. Proceedings [...]. Zurick: ICBEN, 2017. p. 16-29. Disponível em: http://www.icben.org/2017/index.html

DELLA CROCIATA, S; MARTELLOTTA, F.; SIMONE, A. A measurement procedure to assess indoor environment quality for hypermarket workers. Building and Environment, v. 47, pp. 288-299, 2012.

DOI:https://doi.org/10.1016/j.buildenv.2011.07.011 
DELLA CROCIATA, S.; SIMONE, A.; MARTELLOTTA, F. Acoustic comfort evaluation for hypermarket workers. Building and Environment, v. 59, p. 369-378, 2013. DOI: https://doi.org/10.1016/j.buildenv.2012.09.002

EBISSOU, A.; PARIZET, E.; CHEVRET, P. Use of the Speech Transmission Index for the assessment of sound annoyance in open-plan offices. Applied Acoustics, v. 88, p. 90-95, 2015.

DOI:https://doi.org/10.1016/j.apacoust.2014.07.012

EGAN, M. D. Architectural Acoustics. United States of America: McGraw-Hill, 1988.

EVANS, G. W.; JOHNSON, D. Stress and Open-Office Noise. Journal of Applied Psychology, Michigan, v. 85, n. 5, p. 779-783, out. 2000. DOI: https://doi.org/10.1037/0021-9010.85.5.779

GALBRUN, L.; KITAPCI, K. Accuracy of speech transmission index predictions based on the reverberation time and signal-to-noise ratio. Applied Acoustics, v. 81, pp. 1-14, 2014. DOI: https://doi.org/10.1016/j.apacoust.2014.02.001

GOLMOHAMMADI, R.; ALIABADI, M.; NEZAMI, T. An Experimental Study of Acoustic Comfort in Open Space Banks Based on Speech Intelligibility and Noise Annoyance Measures. Archives of Acoustics, v. 42, n. 2, p. 333-347, 2017. DOI: http://dx.doi.org/10.1515\%2Faoa-2017-0035

GONZÁLEZ, C. Evaluación del ruido en ergonomía: criterio RC MARK II. Instituto Nacional de Seguridad e Higiene en el Trabajo - Notas Técnicas de Prevención. Madrid, 2008.

HAAPAKANGAS, A. et al. Effects of unattended speech on performance and subjective distraction: The role of acoustic design in open-plan offices. Applied Acoustics, v. 86, p. 1-16, 2014.

DOI:https://doi.org/10.1016/j.apacoust.2014.04.018

HAKA, M. et al. Performance effects and subjective disturbance of speech in acoustically different office types-a laboratory experiment. Indoor Air, v. 19, p. 454-467, 2009. DOI: https://doi.org/10.1111/j.1600-0668.2009.00608.x

HEDGE, A. The Open-Plan Office: a systematic investigation of employee reaction of their work environment. Environment and Behavior, v. 14, n. 5, p. 519-542, 1982. DOI: https://doi.org/10.1177\%2F0013916582145002

HODGSON, M.; NOSAL, E. M. Effect of noise and occupancy on optimal reverberation times for speech intelligibility in classrooms. The Journal of the Acoustical Society of America, v. 111, n. 2, pp. 931-939, 2002.

DOI:https://doi.org/10.1121/1.1428264

HONGISTO, V. et al. Acoustic satisfaction in an open-plan office before and after the renovation. In: EUROPEAN CONGRESS AND EXPOSITION ON NOISE CONTROL ENGINEERING, 2012, Prague. Proceedings [...]. Prage: European Acoustics Association \& Czech Acoustical Association, 2012. p. 654-659.

HONGISTO, V. et al. Work performance in private office rooms: The effects of sound insulation and sound masking. Building and Environment, v. 104, p. 263-274, 2016. DOI: https://doi.org/10.1016/j.buildenv.2016.04.022

HOUTGAST, T.; STEENEKEN, H. J. M. The modulation transfer function in room acoustics. Technical Review. Bruel \& Kjaer an HBK company, v. 3, p. 3-12, 1985.

HUANG, L. et al. A study on effects of thermals, luminous, and acoustic environments on indoor environmental comfort in offices. Building and Environment, v. 49, p. 304-309, 2011.

DOI:https://doi.org/10.1016/j.buildenv.2011.07.022

IEC - INTERNATIONAL ELECTROTECHNICAL COMMISSION. ICE 60268-16: International Standard: Sound system equipment - Part 16: Objective rating of speech intelligibility by speech transmission index. Switzerland, 2011.

ISO - INTERNATIONAL ORGANIZATION FOR STANDARDIZATION. ISO 3382-2: Acoustics - Measurement of room acoustic parameters - Part 2: Reverberation time in ordinary rooms. Suíça, 2008. 
ISO - INTERNATIONAL ORGANIZATION FOR STANDARDIZATION. ISO 3382-3: Acoustics - Measurement of room acoustic parameters - Part 3: Open plan Offices. Suíça, 2012.

ISO - INTERNATIONAL ORGANIZATION FOR STANDARDIZATION. ISO 9921-1. Ergonomic assessment of speech communication -- Part 1: Speech interference level and communication distances for persons with normal hearing capacity in direct communication (SIL method). Suiça, 1996a.

ISO - INTERNATIONAL ORGANIZATION FOR STANDARDIZATION. ISO 11690-1: Acoustics - Recommended practice for the design of low-noise workplaces containing machinery - Part 1: Noise control strategies. Suiça, $1996 \mathrm{~b}$.

JAHNCKE, H.; HONGISTO, V.; VIRJONEN, P. Cognitive performance during irrelevant speech: Effects of speech intelligibility and office-task characteristics. Applied Acoustics, v. 74, p. 307-316, 2013.

DOI:https://doi.org/10.1016/j.apacoust.2012.08.007

KAARLELA-TUOMAALA, A. et al. Effects of acoustic environment on work in private office rooms and open-plan offices - longitudinal study during relocation. Ergonomics, v. 52, p. 1423-1444, 2009.

DOI:https://doi.org/10.1080/00140130903154579

KERANEN, J.; HONGISTO, V. Prediction of the spatial decay of speech in open-plan offices. Applied Acoustics, v. 74, p. 1315-1325, 2013. DOI: https://doi.org/10.1016/j.apacoust.2013.05.011

KIM, J.; DEAR, R. D. Workspace satisfaction: The privacy-communication trade-off in open-plan offices. Journal of Environmental Psychology, v. 36, p. 18-26, 2013. DOI: https://doi.org/10.1016/j.jenvp.2013.06.007

KITAPCI, K. et al. Speech intelligibility in multilingual spaces. In: EUROPEAN CONGRESS AND EXPOSITION ON NOISE CONTROL ENGINEERING, 2013, Innsbruck. Proceedings [...]. Innsbruck: European Acoustics Association \& Austrian Noise Abatement Association, 2013.

LEE, Y.S. Office layout affecting privacy, interaction, and acoustic quality in LEED-certified buildings. Building and Environment, v. 45, p. 1594-1600, 2010. DOI: https://doi.org/10.1016/j.buildenv.2010.01.007

LIU, T. et al. Effects of noise type, noise intensity, and illumination intensity on reading performance. Applied Acoustics, v. 120, p. 70-74, 2017. DOI: https://doi.org/10.1016/j.apacoust.2017.01.019

LONG, M. Architectural Acoustics. London: Elsevier, 2011.

MEHTA, M.; JOHNSON, J.; ROCAFORT, J. Architectural acoustics: principles and design. New Jersey, USA. Prentice Hall, 1999.

MÜLLER, S. Medir o STI. In: SEMINÁRIO DE MÚSICA, CIÊNCIA E TECNOLOGIA, 2., 2005, Campinas. Anais [...]. São Paulo: USP, 2005. Disponível em: http://www2.eca.usp.br/smct/ojs/index.php/smct/article/view/20/19

NOWOŚWIAT, A.; OLECHOWSKA, M. Fast estimation of speech transmission index using the reverberation time. Applied Acoustics, v. 102, p. 55-61, 2016. DOI: https://doi.org/10.1016/j.apacoust.2015.09.001

PASSERO, C. R. M.; ZANNIN, P. H. T. O conforto acústico em escritórios panorâmicos: estudo de caso em um escritório real. Ambiente Construído, v. 9, n. 1, p. 93-105, 2009.

PASSERO, C. R. M.; ZANNIN, P. H. T. Acoustic evaluation and adjustment of an open-plan office through architectural design and noise control. Applied Ergonomics, v. 43, p. 1066-1071, $2012 a$.

DOI:https://doi.org/10.1016/j.apergo.2012.03.007

PASSERO, C. R. M.; ZANNIN, P. H. T. Study of the acoustic suitability of an open plan office based on STI and DL2 simulations. Archives of Acoustics, v. 37, n. 2, p. 237-243, 2012b.

PIERRETTE, M. et al. Noise effect on comfort in open-space offices: development of an assessment questionnaire. Ergonomics, v. 58, p. 96-106, 2015. DOI: https://doi.org/10.1080/00140139.2014.961972 
REINTEN, P. et al. The indoor sound environment and human task performance: A literature review on the role of room acoustics. Building and Environment, v. 123, p. 315-332, 2017. DOI:https://doi.org/10.1016/j.buildenv.2017.07.005

RINDEL, J. H. Prediction of acoustical parameters for open plan offices according to ISO 3382-3. The Journal of the Acoustical Society of America, v. 131, n. 4, 2012. DOI: https://doi.org/10.1121/1.4708587

RINDEL, J. H.; CHRISTENSEN, C. L. Acoustical simulation of open-plan offices according to ISO 3382-3. In: EUROPEAN CONGRESS AND EXPOSITION ON NOISE CONTROL ENGINEERING, 2012, Prague. Proceedings [...]. Prage: European Acoustics Association \& Czech Acoustical Association, 2012.

RYHERD, E. E.; WANG, L. M. Implications of human performance and perception under tonal noise conditions on indoor noise criteria. The Journal of the Acoustical Society of America, v. 124, n. 1, pp. 218-226, 2008.

DOI:https://doi.org/10.1121/1.2932075

SELAMAT, F. E.; ZULKIFLI, R. Acoustic comfort in industrial office: a preliminary study at a manufacturing company in malaysia. Malaysian Journal of Public Health Medicine, Special Volume (1), p. 7-14, 2016.

SOMMERHOFF, J.; ROSAS, C. Relación entre los porcentajes de inteligibilidad de pruebas de articulación y valores de STI In: CONGRESSO IBERO-AMERICANO DE ACÚSTICA, 8., 2012, Évora. Anais [...]. Évora: SOBRAC, 2012.

TOCCI, G. C. Room Noise Criteria - The State of the Art in the Year 2000. Noise News International, v. 8, n. 3, p. 106119, 2000.

VAN DE POLL, M. K. et al. Disruption of writing by background speech: The role of speech transmission index. Applied Acoustics, v. 81, pp. 15-18, 2014. DOI: https://doi.org/10.1016/j.apacoust.2014.02.005

VDI - ASSOCIAÇÃO DE ENGENHEIROS ALEMÃES. VDI 2569: Isolamento e design acústico em escritórios. Berlin, 1990.

VENETJOKI, N. et al. The effect of speech and speech intelligibility on task performance. Ergonomics, v. 49, pp. 2068-2091, 2006. DOI: https://doi.org/10.1080/00140130600679142

VIRJONEN, P.; KERÄNEN, J.; HONGISTO, V. Determination of acoustical conditions in open-plan offices: proposal for new measurement method and target values. Acta Acustica united with Acustica, v. 95, p. 279-290, 2009.

DOI:https://doi.org/10.3813/AAA.918150

VISCHER, J. C. O Conceito de conforto ambiental no desempenho do ambiente de trabalho. Ambiente Construído, Porto Alegre, v. 7, n.1, p. 21-34, jan./mar. 2007.

WANG, L. M.; BOWDEN, E. E. Performance review of indoor noise criteria. Architectural Engineering -- Faculty Publications, 9, 2003.

ZHANG, M.; KANG, J; JIAO, F. A social survey on the noise impact in open-plan working environments in China. Science of the Total Environment, v. 438, p. 517-526, 2012. DOI: https://doi.org/10.1016/j.scitotenv.2012.08.082

\footnotetext{
${ }^{1}$ Rodrigo Valdeci Martins

Arquiteto e Urbanista. Mestrado em Arquitetura e Urbanismo pela Universidade Federal de Santa Catarina (UFSC). Endereço postal: Rua Fulgêncio Pereira, Vila Yolanda, Foz do Iguaçu, PR, Brasil. CEP 85853-530.

\section{${ }^{2}$ Erasmo Felipe Vergara Miranda}

Engenheiro Acústico. Doutorado. Universidade Federal de Santa Catarina (UFSC). Professor do Depto. de Engenharia Mecânica, do Programa de Pós-graduação em Engenharia Mecânica (PosMEC) e do Programa de Pósgraduação de Arquitetura e Urbanismo (PósARQ) da UFSC. Endereço postal: Centro Tecnológico - Universidade Federal de Santa Catarina, Caixa Postal 476 - Campus Universitário - Trindade, Florianópolis, SC, Brasil. CEP 88040900 .
} 